\title{
Reproducibility and repeatability of central corneal thickness measurement in healthy eyes using four different optical devices
}

\author{
Remzi Karadag $\cdot$ Murat Unluzeybek $\cdot$ Ozgur Cakici $\odot \cdot$ Ayse Yagmur Kanra $\cdot$ Huseyin Bayramlar
}

Received: 26 April 2016/Accepted: 30 September 2016/Published online: 8 October 2016

(C) The Author(s) 2016. This article is published with open access at Springerlink.com

\begin{abstract}
Aim The aim of the study is to compare the measurements of central corneal thickness (CCT) performed by two examiners with four different methods at different times inter- and intraindividually.

Methods Thirty healthy people were included in the study. In these measurements, an optical low-coherence reflectometry (OLCR), an optic coherence tomography (OCT), a specular microscopy (SM), and a corneal topography (CT) were used. Two examiners performed the measurements in a consecutive manner. After 1-7 days of the first measurements, the second measurements were performed again consecutively. The mean of three measurements was taken in each session for all devices.

Results In OCT measurements, there was a significant difference between two examiners in both sessions $(p<0.001)$, while no significant differences were found between two examiners in first and second sessions in SM, CT, and OLCR measurements. When each examiner's measurements were compared to two
\end{abstract}

R. Karadag $(\square) \cdot$ H. Bayramlar

Department of Ophthalmology, Istanbul Medeniyet University School of Medicine, Goztepe, Istanbul, Turkey e-mail: drrkaradag@yahoo.com

M. Unluzeybek · O. Cakici · A. Y. Kanra Department of Ophthalmology, Istanbul Medeniyet University Goztepe Research and Training Hospital, Goztepe, Istanbul, Turkey sessions, there were no significant differences ( $p>0.05$, for all) except the SM measurements of the first examiner $(p=0.041)$. When the first measurements of two examiners were compared, the smallest values were of OCT. At the first session of two examiners, there was a significant difference between OCT and CT measurements, and between OCT and OLCR $(p<0.001, p=0.002$ for the first examiner and $p<0.001$ for the second examiner, respectively).

Conclusion Our study showed that CCT measurements made by CT and OLCR methods were almost same and highly correlated for both the examiners' measurements. CCTs measured by OCT were on average $30 \mu \mathrm{m}$ thinner than CT and OLCR.

Keywords Central corneal thickness - Pachymetry · Optical low-coherence reflectometry · Optic coherence tomography - Specular microscopy . Corneal topography

\section{Introduction}

Central corneal thickness (CCT) has a great deal of importance in the diagnosis and follow-up of glaucoma and refractive surgical interventions such as cross-linking [1]. Furthermore, measurement of CCT is one of the indirect methods to evaluate whether the cornea endothelium is healthy or not. Therefore, CCT measurement, which can simply be performed through 
various methods, is also used in the follow-up of some diseases such as endothelial dystrophies [2]. Ultrasonic Pachymeter is the gold standard. In this method, however, there are some limitations, such as direct contact of the equipment with the cornea, measurement of the estimated central point, limited definition of the thinnest point of the cornea, and variation according to the individual performing the measurement. Currently, devices such as optical, low-coherence reflectometry, optical coherence tomography, noncontact specular microscopy, and corneal topography have been developed to measure CCT with a noncontact optic method [3]. These noncontact methods are less dependent on the operator and have the advantage of demonstrating the thinnest parts of the cornea. Thus, the reliability of the CCT measurements is very important in the diagnosis and management of various corneal diseases [2].

The aim of this study is to compare the measurements and operating individuals in terms of measurement values obtained in four different noncontact methods of CCT measurement performed by two different individuals, to compare the measurements they perform in different time periods, and to evaluate whether these methods can be interchangeable or not.

\section{Materials and methods}

Thirty individuals who applied to our clinic were included in the study. All cases were informed about the study, and informed consent was obtained. This study was performed in accordance with the Helsinki Declaration criteria. Thirty right eyes of 14 male and 16 female patients without any systemic diseases and aged between 22 and 60 years were included in the study. Patients with prior ocular surgery, the presence of glaucoma, refractive errors greater than \pm 2 diopters, cataracts, corneal opacity, and keratoconus were excluded from the study.

Optical low-coherence reflectometry (OLCR; LenStar LS900; Haag-Streit AG, Koeniz, Switzerland), optical coherence tomography (OCT; Topcon 3D OCT-2000, Topcon Medical System, Oakland, New Jersey, USA), noncontact Specular Microscopy (SM; Topcon SP-3000P; Topcon Corporation, Tokyo, Japan), and corneal topography (CT; Sirius; Costruzione Strumenti Oftalmici, Florence, Italy) equipments were used to measure CCT.
Corneal thickness measurements were performed by two individuals consecutively. One to seven days after the first measurement, second measurements were performed in all patients by the same two examiners. Measurements were performed between 10 a.m. and 2 p.m. to avoid diurnal variations. Three measurements were performed on each piece of equipment, and the means were calculated.

SPSS 16 program was used in the statistical analysis, and parametric tests that were used for the distribution of the data were normal. The level of significance was accepted as $p<0.05$.

\section{Results}

Fourteen males and 16 females, with a mean age of $30.97 \pm 7.75$ years, were included in the study. The results of the first and second measurements performed by the first and second investigators are shown in Table 1. A statistically significant correlation was present between the first and second measurements conducted by both investigators in all equipments (in all: $p<0.001)$. A powerful correlation was found between the measurements of the two sessions in the SM, CT, and OLCR methods, while a correlation of an intermediate level was present between the measurements performed using OCT equipment $(r: 0.747, r$ : 0.691 , Table 1 , respectively).

In terms of the measurements of the same individual in both sessions, no significant differences were found between any first and second session measurements of the same individual $(p>0.05$ for all, Table 2), except the SM measurements of the first investigator $(p=0.041)$.

When the two investigators were compared with each other based on the measurements obtained, the measurements by both investigators using SM, CT, and OLCR were similar (in all: $p>0.05$ ); however, OCT measurements by the two investigators were different from each other, both in the first $(p<0.001)$ and second sessions $(p<0.001)$ (Table 3$)$. When the first and second session measurements performed using different types of equipment were compared to each other, the difference between the two sessions was statistically significant in the OCT measurements, but no difference was found in the two measurements in other equipment (Table 3). SM, CT, and OLCR measurements demonstrated a powerful correlation between the two 
Table 1 Correlation between researchers of both the first and second measurements in the same device ( $r$ : Pearson correlation coefficient)

\begin{tabular}{lllll}
\hline Methods & First session $(\mu \mathrm{m})$ & Second session $(\mu \mathrm{m})$ & $r$ value & $p$ value \\
\hline First examiner & & & & \\
OCT & $514.18 \pm 25.60$ & $512.60 \pm 20.85$ & 0.747 & $<0.001$ \\
Specular microscopy & $525.54 \pm 34.64$ & $523.21 \pm 34.51$ & 0.985 & $<0.001$ \\
Corneal topography & $547.46 \pm 32.96$ & $546.61 \pm 31.44$ & 0.989 & $<0.001$ \\
OLCR & $544.25 \pm 31.16$ & $544.64 \pm 30.90$ & 0.987 & $<0.001$ \\
Second examiner & & & & \\
OCT & $502.06 \pm 22.33$ & $499.24 \pm 22.29$ & 0.691 & $<0.001$ \\
Specular microscopy & $524.53 \pm 34.60$ & $524.13 \pm 33.81$ & 0.988 & $<0.001$ \\
Corneal topography & $546.69 \pm 32.14$ & $546.12 \pm 31.22$ & 0.989 & $<0.001$ \\
OLCR & $544.81 \pm 30.96$ & $544.45 \pm 32.49$ & 0.986 & $<0.001$ \\
\hline
\end{tabular}

Table 2 Statistical differences between researchers of both the first and second measurement values of the same unit

\begin{tabular}{lccc}
\hline Devices & First session mean (range) & Second session mean (range) & $p$ value \\
\hline First examiner $(\mu \mathrm{m})$ & & & \\
OCT & $514.18 \pm 25.60(455.67-553.00)$ & $512.60 \pm 20.85(469.00-548.00)$ & 0.617 \\
Specular microscopy & $525.54 \pm 34.64(462.33-579.00)$ & $523.21 \pm 34.51(460.00-573.00)$ & $\mathbf{0 . 0 4 1}$ \\
Corneal topography & $547.46 \pm 32.96(497.33-602.33)$ & $546.61 \pm 31.44(492.00-600.00)$ & 0.355 \\
OLCR & $544.25 \pm 31.16(494.33-594.00)$ & $544.64 \pm 30.90(491.67-597.33)$ & 0.672 \\
Second examiner & $502.06 \pm 22.33(459.33-546.67)$ & $499.24 \pm 22.29(460.67-551.33)$ \\
OCT & $524.53 \pm 34.60(466.00-581.00)$ & $524.13 \pm 33.81(456.67-573.67)$ \\
Specular microscopy & $546.69 \pm 32.14(492.33-597.33)$ & $546.12 \pm 31.22(494.67-593.67)$ \\
Corneal topography & $544.81 \pm 30.96(494.00-598.00)$ & $544.45 \pm 32.49(486.33-592.00)$ \\
OLCR & $14.37 \pm 9.52(0.33-35.00)$ & & 0.387 \\
Differences between two examiners & & 0.689 \\
OCT & $3.86 \pm 4.30(0.00-20.00)$ & $3.30 \pm 2.52(0.33-10.34)$ & 0.716 \\
Specular microscopy & $2.81 \pm 2.35(0.00-9.34)$ & $2.60 \pm 1.99(0.00-6.66)$ & 0.759 \\
Corneal topography & $2.80 \pm 2.29(0.00-9.00)$ & $3.58 \pm 3.59(0.00-16.66)$ & 0.474 \\
OLCR & &
\end{tabular}

Bold values are statistically significant measurements

$O C T$ optic coherence tomography, OLCR optical low-coherence reflectometry

investigators in both sessions. OCT measurements by the two investigators were highly correlated in the first session ( $r$ : 0.876) and intermediately correlated in the second session ( $r$ : 0.668) (Table 4).

A significant difference was also found between the OCT measurements and CT and OLCR measurements of the first session by both investigators (for the measurements of the first investigator: $p<0.001$, $p=0.002$ and for the measurements of second investigator $p<0.001, p<0.001$, respectively).

The measurements of the first investigator using OCT and SM equipment demonstrated no difference $(p=0.972)$, except for between the measurements of the second investigator $(p=0.029)$, (Table 5).
When the SM and CT measurements by both investigators were compared, significant differences were found between the measurements $(p=0.046$ and $p=0.033$ for the first and second investigators, respectively (Table 5). No significant difference was found between the SM and OLCR measurements ( $p=0.134$ for the first investigator, $p=0.066$ for the second investigator) (Table 5). No significant differences were also found between the CT and OLCR measurements by both investigators $(p=1.0)$ (Table 5).

When the correlations of the results of the different methods were analyzed, the first measurements by both investigators were statistically significant (in all: $p<0.001)$ (Table 6). 
Table 3 Statistical differences between the two session's measurement values of the examiners in the same method

\begin{tabular}{|c|c|c|c|}
\hline Methods & First examiner mean (range) & Second examiner mean (range) & $p$ value \\
\hline \multicolumn{4}{|l|}{ First session $(\mu \mathrm{m})$} \\
\hline OCT & $514.18 \pm 25.60(455.67-553.00)$ & $502.06 \pm 22.33(459.33-546.67)$ & $<0.001$ \\
\hline Specular microscopy & $525.54 \pm 34.64(462.33-579.00)$ & $524.53 \pm 34.60(466.00-581.00)$ & 0.341 \\
\hline Corneal topography & $547.46 \pm 32.96(497.33-602.33)$ & $546.69 \pm 32.14(492.33-597.33)$ & 0.255 \\
\hline OLCR & $544.25 \pm 31.16(494.33-594.00)$ & $544.81 \pm 30.96(494.00-598.00)$ & 0.405 \\
\hline \multicolumn{4}{|l|}{ Second session $(\mu \mathrm{m})$} \\
\hline OCT & $512.60 \pm 20.85(469.00-548.00)$ & $499.24 \pm 22.29(460.67-551.33)$ & $<0.001$ \\
\hline Specular microscopy & $523.21 \pm 34.51(460.00-573.00)$ & $524.13 \pm 33.81(456.67-573.67)$ & 0.227 \\
\hline Corneal topography & $546.61 \pm 31.44(492.00-600.00)$ & $546.12 \pm 31.22(494.67-593.67)$ & 0.419 \\
\hline OLCR & $544.64 \pm 30.90(491.67-597.33)$ & $544.45 \pm 32.49(486.33-592.00)$ & 0.832 \\
\hline \multicolumn{4}{|c|}{ Differences between two seasons } \\
\hline OCT & $13.15 \pm 10.80(0.00-38.00)$ & $12.32 \pm 12.61(0.00-51.33)$ & 0.349 \\
\hline Specular microscopy & $4.71 \pm 4.29(1.00-19.67)$ & $3.93 \pm 3.66(0.00-16.66)$ & 0.531 \\
\hline Corneal topography & $3.89 \pm 3.01(0.00-12.33)$ & $3.63 \pm 3.01(0.33-15.34)$ & 0.721 \\
\hline OLCR & $3.85 \pm 3.13(0.34-12.33)$ & $3.90 \pm 3.76(0.33-17.34)$ & 0.302 \\
\hline
\end{tabular}

Bold values are statistically significant measurements

$O C T$ optic coherence tomography, OLCR optical low-coherence reflectometry

Table 4 Correlation between both session to the values of the researchers in the same device ( $r$ : Pearson correlation coefficient)

OCT optic coherence tomography, OLCR optical low-coherence reflectometry

\begin{tabular}{lcccc}
\hline Methods & First examiner & Second examiner & $r$ value & $p$ value \\
\hline First session $(\mu \mathrm{m})$ & & & & \\
OCT & $514.18 \pm 25.60$ & $502.06 \pm 22.33$ & 0.876 & $<0.001$ \\
Specular microscopy & $525.54 \pm 34.64$ & $524.53 \pm 34.60$ & 0.986 & $<0.001$ \\
Corneal topography & $547.46 \pm 32.96$ & $546.69 \pm 32.14$ & 0.994 & $<0.001$ \\
OLCR & $544.25 \pm 31.16$ & $544.81 \pm 30.96$ & 0.993 & $<0.001$ \\
Second session $(\mu \mathrm{m})$ & & & & \\
OCT & $512.60 \pm 20.85$ & $499.24 \pm 22.29$ & 0.668 & $<0.001$ \\
Specular microscopy & $523.21 \pm 34.51$ & $524.13 \pm 33.81$ & 0.993 & $<0.001$ \\
Corneal topography & $546.61 \pm 31.44$ & $546.12 \pm 31.22$ & 0.995 & $<0.001$ \\
OLCR & $544.64 \pm 30.90$ & $544.45 \pm 32.49$ & 0.988 & $<0.001$ \\
\hline
\end{tabular}

The results of SM, CT, and OLCR measurements were highly correlated with each other, while the results of OCT were intermediately correlated with the other three methods (Table 6).

\section{Discussion}

Until recently, the single method used in the measurement of corneal thickness was an ultrasoundbased method. However, this method has some disadvantages such as relatively variable results due to the difficulties in centralization and accommodation, the necessity for topical anesthesia, the possibility of epithelial damage due to contact between the probe and the cornea, and the risk of infection [4, 5]. CCT was reported to show a $\pm 10-\mu \mathrm{m}$ variation following topical anesthesia application $[6,7]$.

Advances in the diagnostic technologies and increased interest in corneal refractive surgical techniques resulted in the development of less invasive and noncontact methods for the measurement of the corneal thickness [8]. Since CCT measurements might be performed using many types of noncontact 
Table 5 A comparison (P values) of the first session of the researchers measurements (Bonferroni multiple comparison)

\begin{tabular}{lrrr}
\hline Devices & OCT & Specular microscopy & Corneal topography \\
\hline First examiner & & & OLCR \\
OCT $(514.18 \pm 25.60 \mu \mathrm{m})$ & & 0.972 & $<0.001$ \\
Specular microscopy $(525.54 \pm 34.64 \mu \mathrm{m})$ & 0.972 & & 0.046 \\
Corneal topography $(547.46 \pm 32.96 \mu \mathrm{m})$ & $<0.001$ & 0.046 & 1.000 \\
OLCR $(544.25 \pm 31.16 \mu \mathrm{m})$ & 0.002 & 0.134 & 0.134 \\
Second examiner & & & $<0.001$ \\
OCT $(502.06 \pm 22.33 \mu \mathrm{m})$ & & 0.029 & 0.033 \\
Specular microscopy $(524.53 \pm 34.60 \mu \mathrm{m})$ & 0.029 & & 1.000 \\
Corneal topography $(546.69 \pm 32.14 \mu \mathrm{m})$ & $<0.001$ & 0.033 & 1.000 \\
OLCR $(544.81 \pm 30.96 \mu \mathrm{m})$ & $<0.001$ & 0.066 & 0.066 \\
\hline
\end{tabular}

OCT optic coherence tomography, OLCR optical low-coherence reflectometry

Table 6 The researchers compared in each of the first measurements of all devices (r: Pearson Correlation Coefficient)

\begin{tabular}{|c|c|c|c|c|c|c|c|c|}
\hline \multirow[t]{2}{*}{ Devices } & \multicolumn{2}{|l|}{ OCT } & \multicolumn{2}{|c|}{ Specular microscopy } & \multicolumn{2}{|c|}{ Corneal topography } & \multicolumn{2}{|l|}{ OLCR } \\
\hline & $r$ & $p$ & $r$ & $p$ & $r$ & $p$ & $r$ & $p$ \\
\hline \multicolumn{9}{|l|}{ First researcher } \\
\hline OCT & & & 0.655 & $<0.001$ & 0.627 & $<0.001$ & 0.644 & $<0.001$ \\
\hline Specular microscopy & 0.655 & $<0.001$ & & & 0.966 & $<0.001$ & 0.974 & $<0.001$ \\
\hline Corneal topography & 0.627 & $<0.001$ & 0.966 & $<0.001$ & & & 0.984 & $<0.001$ \\
\hline OLCR & 0.644 & $<0.001$ & 0.974 & $<0.001$ & 0.984 & $<0.001$ & & \\
\hline \multicolumn{9}{|l|}{ Second researcher } \\
\hline OCT & & & 0.654 & $<0.001$ & 0.678 & $<0.001$ & 0.632 & $<0.001$ \\
\hline Specular microscopy & 0.654 & $<0.001$ & & & 0.976 & $<0.001$ & 0.984 & $<0.001$ \\
\hline Corneal topography & 0.678 & $<0.001$ & 0.976 & $<0.001$ & & & 0.980 & $<0.001$ \\
\hline OLCR & 0.632 & $<0.001$ & 0.984 & $<0.001$ & 0.980 & $<0.001$ & & \\
\hline
\end{tabular}

OCT optic coherence tomography, OLCR optical low-coherence reflectometry

equipment, different types of equipments are available in different clinics. For the follow-up of the patients, a correlation of the results of the measurements performed by the equipment among each other and within a single piece of equipment is still important.

In the study of Borrego-Sanz L et al. which included 76 healthy patients using Pentacam, Ultrasound Pachymetry, Specular Microscopy, and Optic Biometer Lenstar LS 900 equipment, the CCT measurement results were reported as $565.36 \pm 36.34$, $549.61 \pm 32.64, \quad 537.24 \pm 36.34, \quad$ and $443.06 \pm$ $34.28 \mu \mathrm{m}$, respectively. They found the highest correlation between Lenstar and UP [18]. Similarly, Tai et al. also found a high correlation between Lenstar and UP in their study that was performed on 184 eyes [9]. O'Donnell et al. [10] and Zhao et al. [11] found a high correlation between CCT measurements performed by Pentacam and Lenstar; however, they reported that these two devices could not be used in place of each other in clinical applications. Although a good correlation has been reported in UP and Pentacam in many studies, Tai et al. [19] found that Pentacam values were $10 \mu \mathrm{m}$ higher than the UP values. They suggested such a difference may have originated from the fact that lacrima is also measured during measurement by Pentacam, and that UP 
applies pressure on the cornea. Moreover, Almubrad et al. [6] reported that CCT values measured by SM were lower than the values of UP $(28.17 \pm$ $19.20 \mu \mathrm{m})$, and they concluded that the two methods could not be used in place of each other.

Huang et al. [12] measured CCT in 66 healthy corneas and reported the results as follows: $538.82 \pm 26.46 \mu \mathrm{m}$ with Pentacam, 542.14 \pm $27.12 \mu \mathrm{m}$ with Sirius, $548.10 \pm 26.41 \mu \mathrm{m}$ with Galilei, and $532.81 \pm 26.24 \mu \mathrm{m}$ with RTVue. Repeatability and reproducibility were very high in these four devices, and it was suggested to be adequate for clinical use. Although the measurements of these devices were not correlated with each other since they were different, they emphasized that Galilei yielded better results. Additionally, using similar optic methods (Pentacam and RTVue), Nam et al. [13] and Chen et al. [14] achieved high intraobserver repeatability and interobserver reproducibility.

Since there are no studies of intra- and interobserver comparisons using the equipment we used in this study, it was not possible to perform a one-to-one comparison with other studies. The present study revealed that interobserver repeatability of OCT was poor, and the differences between the measurements were significant. Interobserver repeatability of other methods was good. We found interobserver and intraobserver repeatability of the measurements performed by OLCR and CT devices, and this value was less than $1 \mu \mathrm{m}$.

Milla et al. [15] and Savini et al. [16] found high repeatability in CCT measurements performed by Sirius and Galilei in the same order. Also, Khoramnia et al. [17] and Milla et al. [15] reported good repeatability in the same order using Pentacam and Sirius. Savini et al. [18] found the repeatability of Sirius slightly poorer than Galilei and slightly better than Pentacam. Many investigators [19, 20] found CCT measurement repeatability of Pentacam to be poorer compared to FD-OCT. A probable cause of this was reported to be the more rapid screening capacity of OCT.

In the present study, the results of measurements by OCT were lower than the values obtained with other equipment. Furthermore, while OCT measurement results were intermediate-high correlated with the results of other equipment, the results of other equipment were highly correlated with each other.
Current and some previous studies have suggested that different devices for CCT evaluation cannot be used in place of each other [21-23]. However, there are also studies reporting correlations from intermediate to excellent levels between Galilei and Orbscan II, and between Pentacam and Orbscan II [16, 24, 25]. In one study, the results of Orbscan II were markedly lower than the measurements performed using Pentacam and Galilei [26]. On the contrary, Sedaghat et al. [27] found the CCT values measured with Orbscan II to be slightly higher than the values obtained using Pentacam. Bueh et al. found no significant differences between the measurements of these two pieces of equipment [28]. However, in another study by Amano et al., no significant difference was found between the measurements of Orbscan II and Pentacam [29]. Kim et al. found that the CCT measurements prior to photorefractive keratectomy by Orbscan II in myopic patients were similar with the results of Pentacam; however, they reported that postoperative early and late measurements by Orbscan II resulted in thinner values [30].

In conclusion, values obtained in the present study by CT and OLCR methods taken by different individuals and the measurements obtained by the same individuals were almost similar and highly correlated. The difference between the results of these two measurement methods was approximately $1 \mu \mathrm{m}$. Since a significant difference was present in the results of the measurements of two different individuals performed by OCT, the reliability of this method in interobserver measurements in the evaluation of CCT is lower compared to the other methods according to our study. In addition, OCT measurements yield the lowest values compared to the other methods. We consider that the presence of a difference of at least $30 \mu \mathrm{m}$ between the measurement values of CT and OCLR, and measurements of OCT will affect the calculations for corrected intraocular pressure and plan the operations in refractive surgery.

\section{Compliance with ethical standards}

Conflict of interest None of the authors has any conflicts of interest in this study.

Open Access This article is distributed under the terms of the Creative Commons Attribution 4.0 International License (http:// creativecommons.org/licenses/by/4.0/), which permits unrestricted use, distribution, and reproduction in any medium, provided you give appropriate credit to the original author(s) and the source, provide a link to the Creative Commons license, and indicate if changes were made. 


\section{References}

1. Marsich MW, Bullimore MA (2000) The repeatability of corneal thickness measures. Cornea 19:792-795

2. Gherghel D, Hosking SL, Mantry S et al (2004) Corneal pachymetry in normal and keratoconic eyes: Orbscan II versus ultrasound. J Cataract Refract Surg 30:1272-1277

3. Gonul S, Koktekir BE, Bakbak B et al (2014) Comparison of central corneal thickness measurements using optical lowcoherence reflectometry, Fourier domain optical coherence tomography, and Scheimpflug camera. Arq Bras Oftalmol 77:345-350

4. Kawana K, Tokunaga T, Miyata K et al (2004) Comparison of corneal thickness measurements using Orbscan II, noncontact specular microscopy, and ultrasonic pachymetry in eyes after laser in situ keratomileusis. $\mathrm{Br} \mathbf{J}$ Ophthalmol 88:466-468

5. Cakici O (2014) Clinical significance of central corneal thickness and comparison of central corneal thickness measurements methods. J Clin Exp Invest 5:153-158

6. Almubrad TM, Osuagwu UL, Abbadi IA et al (2011) Comparison of the precision of the Topcon SP-3000P specular microscope and an ultrasound pachymeter. Clin Ophthalmol 5:871-876

7. Asensio I, Rahhal SM, Alonso L et al (2003) Corneal thickness values before and after oxybuprocaine $0.4 \%$ eye drops. Cornea 22:527-532

8. Borrego-Sanz L, Saenz-Frances F, Bermudez-Vallecilla M et al (2014) Agreement between central corneal thickness measured using Pentacam, ultrasound pachymetry, specular microscopy and optic biometer Lenstar LS 900 and the influence of intraocular pressure. Ophthalmologica 231:226-235

9. Tai LY, Khaw KT, Ng CM et al (2013) Central corneal thickness measurements with different imaging devices and ultrasound pachymetry. Cornea 32:766-771

10. O'Donnell C, Hartwig A, Radhakrishnan H (2012) Comparison of central corneal thickness and anterior chamber depth measured using LenStar LS900, Pentacam, and Visante AS-OCT. Cornea 31:983-988

11. Zhao J, Chen Z, Zhou Z et al (2013) Evaluation of the repeatability of the Lenstar and comparison with two other non-contact biometric devices in myopes. Clin Exp Optom 96:92-99

12. Huang J, Ding X, Savini G et al (2013) A comparison between Scheimpflug imaging and optical coherence tomography in measuring corneal thickness. Ophthalmology 120:1951-1958

13. Nam SM, Im CY, Lee HK et al (2010) Accuracy of RTVue optical coherence tomography, Pentacam, and ultrasonic pachymetry for the measurement of central corneal thickness. Ophthalmology 117:2096-2103

14. Chen S, Huang J, Wen D et al (2012) Measurement of central corneal thickness by high-resolution Scheimpflug imaging, Fourier-domain optical coherence tomography and ultrasound pachymetry. Acta Ophthalmol 90:449-455

15. Milla M, Pinero DP, Amparo F et al (2011) Pachymetric measurements with a new Scheimpflug photography-based system; intraobserver repeatability and agreement with optical coherence tomography pachymetry. J Cataract Refract Surg 37:310-316
16. Savini G, Carbonelli M, Barboni P et al (2011) Repeatability of automatic measurements performed by a dual Scheimpflug analyzer in unoperated and post-refractive surgery eyes. J Cataract Refract Surg 37:302-309

17. Khoramnia R, Rabsilber TM, Auffarth GU (2007) Central and peripheral pachymetry measurements according to age using the Pentacam rotating Scheimpflug camera. J Cataract Refract Surg 33:830-836

18. Savini G, Barboni P, Carbonelli M et al (2011) Repeatability of automatic measurements by a new Scheimpflug camera combined with Placido topography. J Cataract Refract Surg 37:1809-1816

19. Salouti R, Nowroozzadeh MH, Zamani M et al (2009) Comparison of anterior and posterior elevation map measurements between 2 Scheimpflug imaging systems. J Cataract Refract Surg 35:856-862

20. Shankar H, Pesudovs K (2008) Reliability of peripheral corneal pachymetry with the Oculus Pentacam. J Cataract Refract Surg 34:7

21. Faramarzi A, Karimian F, Jafarinasab MR et al (2010) Central corneal thickness measurements after myopic photorefractive keratectomy using Scheimpflug imaging, scanning-slit topography, and ultrasonic pachymetry. J Cataract Refract Surg 36:1543-1549

22. Lackner B, Schmidinger G, Pieh S et al (2005) Repeatability and reproducibility of central corneal thickness measurement with Pentacam, Orbscan, and ultrasound. Optom Vis Sci 82:892-899

23. Doughty MJ, Jonuscheit S (2010) The Orbscan acoustic (correction) factor for central corneal thickness measures of normal human corneas. Eye Contact Lens 36:106-115

24. Ishibazawa A, Igarashi S, Hanada K et al (2011) Central corneal thickness measurements with Fourier-domain optical coherence tomography versus ultrasonic pachymetry and rotating Scheimpflug camera. Cornea 30:615-619

25. Rao HL, Kumar AU, Kumar A et al (2011) Evaluation of central corneal thickness measurement with RTVue spectral domain optical coherence tomography in normal subjects. Cornea 30:121-126

26. Huang J, Pesudovs K, Yu A et al (2011) A comprehensive comparison of central corneal thickness measurement. Optom Vis Sci 88:940-949

27. Sedaghat MR, Daneshvar R, Kargozar A et al (2010) Comparison of central corneal thickness measurement using ultrasonic pachymetry, rotating Scheimpflug camera, and scanning-slit topography. Am J Ophthalmol 150:780-789

28. Buehl W, Stojanac D, Sacu S et al (2006) Comparison of three methods of measuring corneal thickness and anterior chamber depth. Am J Ophthalmol 141:7-12

29. Amano S, Honda N, Amano Y et al (2006) Comparison of central corneal thickness measurements by rotating Scheimpflug camera, ultrasonic pachymetry, and scanningslit corneal topography. Ophthalmology 113:937-941

30. Kim SW, Byun YJ, Kim EK et al (2007) Central corneal thickness measurements in unoperated eyes and eyes after PRK for myopia using Pentacam, Orbscan II, and ultrasonic pachymetry. J Refract Surg 23:888-894 